\title{
Chemical Modulators of Fibrinogen Production and Their Impact on Venous Thrombosis
}

\author{
Rui Vilar $^{1}$ Samuel W. Lukowski ${ }^{1,2}$ Marco Garieri ${ }^{1}$ Corinne Di Sanza ${ }^{1}$ Marguerite Neerman-Arbez ${ }^{1,3}$ \\ Richard J. Fish ${ }^{1}$
}

${ }^{1}$ Department of Genetic Medicine and Development, University of Geneva Faculty of Medicine, Geneva, Switzerland

2 Institute for Molecular Bioscience, The University of Queensland, Saint Lucia, Queensland, Australia

3 iGE3, Institute of Genetics and Genomics in Geneva, Geneva, Switzerland

\begin{abstract}
Address for correspondence Richard J. Fish, Department of Genetic Medicine and Development, Faculty of Medicine, University of Geneva, 1, rue Michel-Servet, Geneva 1206, Switzerland (e-mail: Richard.Fish@unige.ch).
\end{abstract}

Thromb Haemost 2021;121:433-448.

Abstract
Keywords
- all-trans retinoic acid
- anthralin
- fibrinogen
- thrombosis
- zebrafish

Thrombosis is a leading cause of morbidity and mortality. Fibrinogen, the soluble substrate for fibrin-based clotting, has a central role in haemostasis and thrombosis and its plasma concentration correlates with cardiovascular disease event risk and a prothrombotic state in experimental models. We aimed to identify chemical entities capable of changing fibrinogen production and test their impact on experimental thrombosis. A total of 1,280 bioactive compounds were screened for their ability to alter fibrinogen production by hepatocyte-derived cancer cells and a selected panel was tested in zebrafish larvae. Anthralin and all-trans retinoic acid (RA) were identified as fibrinogen-lowering and fibrinogen-increasing moieties, respectively. In zebrafish larvae, anthralin prolonged laser-induced venous occlusion times and reduced thrombocyte accumulation at injury sites. RA had opposite effects. Treatment with RA, a nuclear receptor ligand, increased fibrinogen mRNA levels. Using an antisense morpholino oligonucleotide to deplete zebrafish fibrinogen, we correlated a shortening of laser-induced venous thrombosis times with RA treatment and fibrinogen protein levels. Anthralin had little effect on fibrinogen mRNA in zebrafish larvae, despite leading to lower detectable fibrinogen. Therefore, we made a proteomic scan of anthralin-treated cells and larvae. A reduced representation of proteins linked to the canonical secretory pathway was detected, suggesting that anthralin affects protein secretion. In summary, we found that chemical modulation of fibrinogen levels correlates with measured effects on experimental venous thrombosis and could be investigated as a therapeutic avenue for thrombosis prevention.

\section{Introduction}

Thrombosis is a major contributor to death and disability worldwide, with myocardial infarction, thromboembolic stroke, and venous thromboembolism as key thrombotic cardiovascular disease (CVD) events. ${ }^{1}$ Recognized public awareness of thrombosis is low, ${ }^{2}$ despite its global impact. ${ }^{3}$

CVD event prevention strategies stand as valuable approaches to tackle thrombosis, ${ }^{1}$ but are not without risk $^{4}$ or limitations in terms of therapeutic window, unpredictable dose response, route of administration, the need for frequent monitoring, or limited usefulness..$^{5-8}$ Therefore, identification of novel antithrombotics remains an area of clinical importance. ${ }^{9}$

Hemostasis and thrombosis mechanisms involve numerous factors which can be investigated as potential targets for novel antithrombotic agents using animal models. ${ }^{10}$ As a key clotting protein, fibrinogen is a candidate target in this received

May 8, 2020

accepted after revision

September 2, 2020

published online

December 10, 2020 (c) 2020. Thieme. All rights reserved. Georg Thieme Verlag KG,

Rüdigerstraße 14,

70469 Stuttgart, Germany
DOI https://doi.org/

10.1055/s-0040-1718414. ISSN 0340-6245. 
regard. Fibrinogen circulates as a 340-kDa plasma glycoprotein hexamer with two sets of three polypeptide chains, $(\mathrm{A} \alpha \mathrm{BB} \gamma)_{2}$, contributing to blood coagulation as the soluble precursor to fibrin. Thrombin-mediated cleavage of fibrinopeptides from the $\mathrm{NH}_{2}$-terminus of $\mathrm{A} \alpha$ and $\mathrm{B} \beta$ chains, generate fibrin monomers that polymerize, and form an interconnected fibrin network. Fibrinogen also promotes hemostasis by bridging activated platelets via the $\alpha \operatorname{IIb} \beta 3$ integrin and driving the formation of a platelet plug at sites of injury. ${ }^{11}$

The fibrinogen polypeptides are encoded by three genes, $F G A, F G B$, and $F G G$, clustered on human chromosome 4 (4q32), and co-regulated in a cell-type and chromosomal conformation-dependent manner. ${ }^{12-14}$ Plasma fibrinogen levels are normally maintained within a concentration range of 1.5 to $4 \mathrm{~g} / \mathrm{L}{ }^{11}$ However, high circulating fibrinogen has been associated with CVD event risk in prospective observational studies. ${ }^{15,16}$ These associations are in accordance with functional data from animal models. While hyperfibrinogenemic mice displayed thrombophilia and resistance to thrombolysis, suggesting that elevated fibrinogen can contribute independently and directly to vascular disease, ${ }^{17}$ fibrinogen-deficient mice suffer from spontaneous bleeding and impaired clotting function. ${ }^{18}$ Fibrinogen knockout adult zebrafish also displays a bleeding phenotype ${ }^{19}$ and zebrafish larvae fail to support occlusive venous thrombosis upon laser-induced endothelial injury. ${ }^{20}$

Besides CVD, association of plasma fibrinogen with worsened clinical outcome has been described in other human disorders, ${ }^{21}$ including cancer ${ }^{22,23}$ and neurological disease. ${ }^{24,25}$ It remains unclear if a drug with fibrinogenmodulating capabilities could be used for broader clinical applications.

In the present study, we searched for agents which alter fibrinogen levels in a library of small molecules. Initial screening steps were made with fibrinogen-producing cell lines before functionally testing selected compounds in thrombosis assays in zebrafish larvae. Anthralin lowered fibrinogen production in these settings, whereas all-trans retinoic acid (RA) increased it. The compounds affected thrombocyte adhesionaggregation dynamics and venous thrombosis in a manner which correlated with altered fibrinogen production. While anthralin behaved as an antithrombotic agent, prolonging the time to vessel occlusion and reducing thrombocyte aggregation in venous laser-injury assays, RA had opposite prothrombotic effects. This study identifies agents that alter fibrinogen production with an impact on venous thrombosis, supporting the concept of targeting of plasma fibrinogen as a potential route for thrombosis prevention.

\section{Methods}

\section{Cell Culture}

HepG2 cells were cultured in Minimum Essential Media (Sigma). HuH7 and HEK-293T cells were grown in Dulbecco's Modified Eagle Medium (DMEM, Invitrogen). Media were supplemented with $10 \%$ fetal bovine serum, $100 \mathrm{U} / \mathrm{mL}$ of penicillin, and $100 \mu \mathrm{g} / \mathrm{mL}$ streptomycin (Invitrogen).

\section{Small Molecules}

The Prestwick Chemical Library (Prestwick Chemical, Illkirch, France), comprising 1,280 bioactive small molecules in 96-well plates, was screened for compounds that alter fibrinogen production. After the initial screen and in vitro validation steps, small molecules were purchased in individual vials (Prestwick Chemical).

\section{In Vitro assays}

HepG2 or Huh7 cells were seeded at a density of $2 \times 10^{4}$ cells per well in 96-well plates and incubated for 24 hours at $37^{\circ} \mathrm{C}$, $5 \% \mathrm{CO}_{2}$, before compounds were added in Opti-MEM (Invitrogen). On each plate there were also cells treated with vehicle or media alone. Twenty-four hours posttreatment, secreted fibrinogen present in cell-conditioned media was measured with an enzyme-linked immunosorbent assay, and these values were divided by the total cell-associated protein measured with a small-scale Bradford assay, as described previously. ${ }^{26}$

\section{Chemical Similarity Search}

To assess chemical similarity among compounds affecting fibrinogen production in vitro, we performed an in silico structure clustering analysis with PubChem software that makes use of a single linkage algorithm (https://pubchem. ncbi.nlm.nih.gov/assay/assay.cgi?p=clustering). The software assesses nonhydrogen atoms and by considering intrinsic parameters (e.g., types of atoms in a pair and length of their shortest bond path) computes the Tanimoto coefficient (TC) of similarity. Two structures are typically considered similar if sharing a TC equal to or greater than $0.85 .{ }^{27}$

\section{In Vivo Testing in Zebrafish Embryos}

TU strain Danio rerio was obtained from ZIRC (https://zebrafish.org/home/guide.php), fibrinogen mutants as described previously, ${ }^{19}$ and fish with the itga2b:EGFP transgene (also named cd41:EGFP, a gift from the Zon Laboratory, Harvard Medical School) were maintained under stable aquarium conditions: $26^{\circ} \mathrm{C}, 500 \mu \mathrm{S}$ conductivity, pH 7.5. Experimentation described was authorized by local veterinary authorities. Embryos from natural mating developed at $28.5^{\circ} \mathrm{C}$ in E3 media supplemented with methylene blue (Sigma-Aldrich). Toxicity of compounds was assessed using 3-day postfertilization (dpf) embryos in 24-well plates treated for 2 days at 5 embryos per well, in $1 \mathrm{~mL}$ E3. A range of compound concentrations from mid-nM to mid- $\mu \mathrm{M}$ was tested, three times for each compound. Maximal concentrations that did not visibly affect development and where the larval swim bladder was inflated in $80 \%(4 / 5)$ of embryos at the $5 \mathrm{dpf}$ endpoint were determined. In downstream experiments, when using compounds in $2-3 \mathrm{dpf}$ or $3-5 \mathrm{dpf}$ treatments, larvae were arrayed in 6-well plates, 20 embryos per well, in $5 \mathrm{~mL}$ E3 containing individual chemicals or vehicle (DMSO). The concentration of anthralin $(0.054 \mu \mathrm{M})$ used in 2-3dpf incubations was well tolerated, as seen by normal morphology posttreatment. It exceeds that used for $3-5 \mathrm{dpf}$ incubations $(0.03 \mu \mathrm{M})$ for this reason.

Where described, a morpholino antisense oligonucleotide (MO; GeneTools, LCC), designed to target the fga pre-mRNA 
at the exon 1-intron 1 junction (5'GCATTATATCACTCACCAATGCAGA3') was used. Approximately $1 \mathrm{~nL}$ of a solution containing $6.0,2.0,1.2,1.0$, or $0.8 \mathrm{ng}$ of the morpholino was injected into 1-2 cell-stage embryos prior to treatment with RA or vehicle (DMSO) as described. A morpholino with 5 mismatched nucleotides (5'GCTTAATATGACTCACGAATCCAGA3') was used as a control.

\section{Western Blotting}

3 or 5 dpf zebrafish larvae were homogenized in Tissue Protein Extraction Reagent (T-PER, Invitrogen) containing protease inhibitors (Roche). Supernatants were treated with $2.5 \% \beta$-mercaptoethanol and boiled at $95^{\circ} \mathrm{C}$ for 5 minutes before SDS-PAGE and immunoblotting, essentially as described previously. ${ }^{19}$ Since $A \alpha$ and $B \beta$ chains have similar molecular weight, ${ }^{19}$ in the two zebrafish screening assays we only used antibodies directed against $\mathrm{B} \beta$ and $\gamma$ polypeptides. In turn, in the morpholino experiment, as the synthetic DNAlike molecule was designed to target the fga pre-mRNA, we privileged the use of anti-A $\alpha(E)$ antibodies. Approximately $25 \mathrm{ng}$ of zebrafish fibrinogen, produced in cell culture (see below), was used as a control sample. Immunoreactive bands were imaged using a myECL Imager (Invitrogen) according to the manufacturer's instructions. Membranes were stripped and re-probed with mouse anti- $\beta$-actin antibodies (Sigma) as a representative protein control. Blots were quantified using the open source ImageJ software.

\section{Recombinant Zebrafish Fibrinogen}

The zebrafish fibrinogen cDNAs, fga, fgb, and fgg, were cloned using the pcDNA3.1/V5-His TOPO mammalian expression vector (Invitrogen, Groningen, The Netherlands). Transient transfections of HEK-293T/17 cells that otherwise do not produce fibrinogen were performed using Lipofectamine 2000 reagent (Invitrogen) and a mix of $10 \mu \mathrm{g}$ of each plasmid, according to the manufacturer's instructions. Twelve $15-\mathrm{cm}$ culture dishes were used with $4 \times 10^{6}$ HEK-293T/17 cells seeded per plate the day prior to transfection. Cells were washed with phosphate-buffered saline (PBS) 24 hours after transfection and incubated for 48 hours in serum-free media (Opti-MEM, Invitrogen). The conditioned medium was centrifuged to remove cell debris, harvested, and concentrated to $1 \mathrm{~mL}$ using Amicon Ultra-15, $50 \mathrm{kDa}$ cut-off spin columns (Millipore, Bedford, Massachusetts, United States). Fibrinogen was precipitated using ammonium sulfate. Briefly, the concentrated media was incubated overnight at $4^{\circ} \mathrm{C}$ with $20 \%$ saturated ammonium sulfate. The solution was centrifuged for 15 minutes at $16,100 \mathrm{~g}$, at $4^{\circ} \mathrm{C}$. The pellet was then washed twice with $20 \%$ saturated ammonium sulfate before being resuspended in water at $37^{\circ} \mathrm{C}$.

\section{Reverse Transcription Quantitative Polymerase Chain Reaction}

A total of $3 \times 10^{5}$ HepG2 cells were seeded in 6 -well plates and incubated for 24 hours before treatments. At 48 hours of treatment, RNA was isolated with TRIzol Reagent (Invitrogen). For in vivo experiments, TRIzol was used to isolate RNA from 3 or $5 \mathrm{dpf}$ zebrafish larvae. RNA samples were treated with DNAse (Ambion, AM1907) and reverse transcribed using random hexamer primers and SuperScript II (Invitrogen). Fibrinogen mRNA levels were measured by reverse transcription quantitative polymerase chain reaction (RTqPCR) using a SYBR Green master mix (Applied Biosystems). Expression was normalized to that of $B A C T$ for human cells using oligonucleotides as control templates for quantifica-

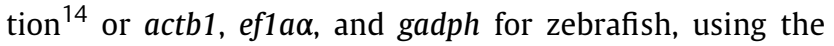
$\Delta \Delta C T$ method. ${ }^{28}$ CT values were obtained using the StepOne software, version 2.3 (Applied Biosystems). Primer sequences were described previously. ${ }^{14,28}$

\section{Zebrafish Blood Vessel Laser Injury Assays}

Two methods were used to investigate the effect of compounds on thrombosis. First, the adhesion and accumulation of fluorescent thrombocytes post venous laser injury were recorded in hemizygous $5 \mathrm{dpf} \operatorname{Tg}($ itga $2 b$ :EGFP) zebrafish larvae after 48 -hour incubations with and without compounds. Second, the TTO of the posterior cardinal vein (PCV) after targeted laser injury was measured in 3dpf larvae after 24hour incubations with compounds. For both assays, larvae were sedated with $0.17 \mathrm{mg} / \mathrm{mL}$ tricaine and placed on a $0.22 \%$ low-gelling temperature agarose (Sigma) in E3 medium, on their right side, prior to targeted laser injury of the PCV near the fifth somite distal to the cloaca. Injuries were made using a Leica Laser Microdissection microscope, enabling targeted laser focusing coupled to an upright DM6500 microscope with a HCX PL FLUOTAR L $20 \times / 0.40$ objective and CC7000 (color) and DFC360FX (black and white) cameras, at room temperature. The laser settings were a constant laser power, amplitude, speed, and target shape. The cryslas laser used has a maximum pulse energy of $50 \mu \mathrm{J}$, a $80 \mathrm{~Hz}$ frequency, and a wavelength of $355 \mathrm{~nm}$. Leica LMD and LAS-AF software were used for data acquisition. MetaMorph software (7.1) was used to quantify thrombocyte accumulation by measuring the kinetics of itga2b:EGFP-associated cell fluorescence accumulation at the injury site. The mean fluorescence measured prior to injury in each recording was subtracted from all data points for that larva. We did not control for potential bleaching effects on EGFP, but saw no evidence for this in the recordings.

\section{Proteome Analysis}

A total of $4.9 \times 10^{6} \mathrm{HepG} 2$ cells were seeded before a 24-hour treatment with $0.9 \mu \mathrm{M}$ anthralin or DMSO (vehicle), detached with trypsin-EDTA, and washed four times with PBS. 3dpf zebrafish embryos were treated for 48 hours with $0.03 \mu \mathrm{M}$ anthralin or vehicle. Samples were lysed using sonication in Rapigest lysis buffer. Proteins were digested with trypsin and peptides analyzed by nanoLC-MSMS using an easy-nLC 1000 (Thermo Fisher Scientific) coupled with a Q-Exactive Plus mass spectrometer (Thermo Fisher Scientific). Database searches were performed with Mascot (Matrix Science) using the Zebrafish Reference Proteome database (Uniprot) or Homo sapiens databases (Uniprot). Data were analyzed and validated with Scaffold (Proteome Software) with a protein and peptide threshold of $95 \%$ and at least one unique peptide per protein. 
Using Scaffold v4.8.7 algorithms, Fisher's exact tests were made for each experiment. Proteins significantly less represented in anthralin-treated samples $(p \leq 0.05)$ compared with control in both experimental replicates (11 and 2 proteins in HepG2 cells and zebrafish larvae, respectively) were selected for enrichment analysis using DAVID v6.8. ${ }^{29,30}$ With only two such proteins, no pathway or domain enrichment was obtained with the zebrafish samples. A second analysis using a nonsignificant threshold $p$-value of 0.31 from the initial Fisher's exact tests was used. This was to look for trends in pathways or domains enriched at this lower p-value stringency in HepG2 cells or zebrafish larvae, compared with those seen in the HepG2 samples at $p \leq 0.05$. This approach allowed us to detect a minimum of 200 proteins less represented in anthralin-treated samples from HepG2 cells or zebrafish larvae, compared with controls. With a $p$ value of up to 0.31 , we do not infer statistical significance from these analyses. In total, 56 and 22 proteins were underrepresented at this $p$-value in replicate HepG2 cell and zebrafish experiments, respectively, and were selected for the enrichment analysis. We report results obtained with the Functional Annotation Chart tool of DAVID. Further details are given in -Supplementary Material and Supplementary Data files (available in the online version).

\section{Data Analysis}

Dose-response curves were calculated by nonlinear regression using the algorithms of Prism 8.2.1 (GraphPad Software, San Diego, California, United States).

\section{Results}

\section{A Small-Molecule Screen for Fibrinogen Modulators}

We screened 1,280 compounds from a bioactive molecule library to identify those with the capacity to alter fibrinogen production in cultured hepatocyte-derived tumor cells (HepG2). The library used has chemical and pharmacological diversity as well as bioavailability and safety data in humans, making compounds amenable to direct entry into an optimization program. ${ }^{31}$

HepG2 cells were incubated with all compounds $(10 \mu \mathrm{M})$ and a ratio of secreted fibrinogen over total cell protein was measured. The screen was completed three times, each with technical triplicates. Data were adjusted for medium alone on all assay plates and normalized to vehicle (DMSO; - Fig. 1A).

Compounds were considered as potential fibrinogen modulators when their production was altered by $>25 \%$, without reducing total protein by more than $30 \%$, to increase the likelihood of selection of molecules with specific effects and avoid compounds with major effects on cell numbers or viability. Approximately $10 \%$ of the drugs (132 compounds) satisfied these criteria; 118 decreased normalized fibrinogen levels in the media (fibrinogen range: $-26 \%$ to $-68 \%$ ); and 14 increased them (fibrinogen range $+25 \%$ to $+115 \%$ ). In total, $90 \%$ of the tested compounds had little or no effect (0-25\%) on fibrinogen production. Nominal $p$-values for fibrinogen in compound-treated versus DMSO were $<0.05$ for 61 and 5 of these compounds ( $n=3$, two-tailed $t$-test), respectively
(-Fig. 1B). Fibrinogen production by HepG2 cells was measured after treatment with these 66 compounds in a range of concentrations $(0.0152-90 \mu \mathrm{M})$. This tested again their ability to alter fibrinogen production and assessed dose-responsiveness and efficacy. A total of 42 compounds gave dosedependent effects (-Fig. 2 and - Supplementary Fig. S1 [available in the online version]), and were subsequently added to cultured Huh7 cells, at $3.3,10$, and $30 \mu \mathrm{M}$, as a second hepatocyte-derived fibrinogen-expressing cellular model. This step aimed to exclude drugs with HepG2 cellspecific fibrinogen-modulating effects. A concentration of $10 \mu \mathrm{M}$ recapitulated the initial screen concentration, and the other concentrations were used to test for dose-responsiveness (-Supplementary Fig. $\mathbf{S 2}$ [available in the online version]). Many of the compounds gave a dose-dependent effect on fibrinogen production in Huh7. However, only 22 were able to alter the fibrinogen production by $\geq 25 \%$ at $10 \mu \mathrm{M}$. These were selected for testing in vivo. Reserpine, a drug that reduced fibrinogen production by $23 \%$ ( $p=0.04$, two-tailed $t$-test), was also included for further experimentation as it had effects that were close to our cut-off criteria.

We searched for structural similarity between compounds affecting fibrinogen production using a PubChem clustering analysis. None of the pairwise comparisons reached a TC of 0.85 , inferring overall structurally dissimilarity. The highest similarities were detected between nocodazole and parbendazole ( $\mathrm{TC}=0.789)$, and carvedilol and reserpine $(\mathrm{TC}=0.729$; - Supplementary Fig. $\mathbf{S 3}$ [available in the online version]). Both nocodazole and parbendazole are agents known to affect the microtubule polymerization dynamics and functions. ${ }^{32,33}$ In turn, carvedilol and reserpine are drugs used to control blood pressure. While the mechanism of action of carvedilol consists in the blockage of $\alpha 1$-receptors of blood vessels, reserpine acts by depleting catecholamines from central and peripheral synapses. ${ }^{34,35}$

\section{Compounds Affect Fibrinogen Production in Zebrafish Larvae}

We used zebrafish larvae to determine if the 23 molecules altering fibrinogen production in vitro could affect it in vivo. This had the convenience of both low-volume drug administration to whole animals and validated follow-up assays of thrombus formation in the conserved vertebrate coagulation system. ${ }^{10}$

As our functional tests of thrombosis use $3 \mathrm{dpf}$ and $5 \mathrm{dpf}$ zebrafish larvae (see below), we looked for small moleculeinduced changes in fibrinogen levels at these developmental ages, starting with 5dpf embryos treated for 48 hours (-Fig. 3A). Maximum doses presenting no signs of toxic effects on embryonic development for each drug were used. Whole larvae fibrinogen protein levels were assessed by the quantification of fibrinogen $\mathrm{B} \beta$ chain immunoblotting, using $\beta$-actin for normalization. All-trans RA treatment increased fibrinogen levels by $>80 \%$. Incubation of larvae with anthralin and loteprednol etabonate (LE) decreased fibrinogen production by 31 and 60\%, respectively (-Figs. 3B-F).

RA, LE, and anthralin were then tested for their effects on fibrinogen production in younger zebrafish embryos, using a 


\section{A}
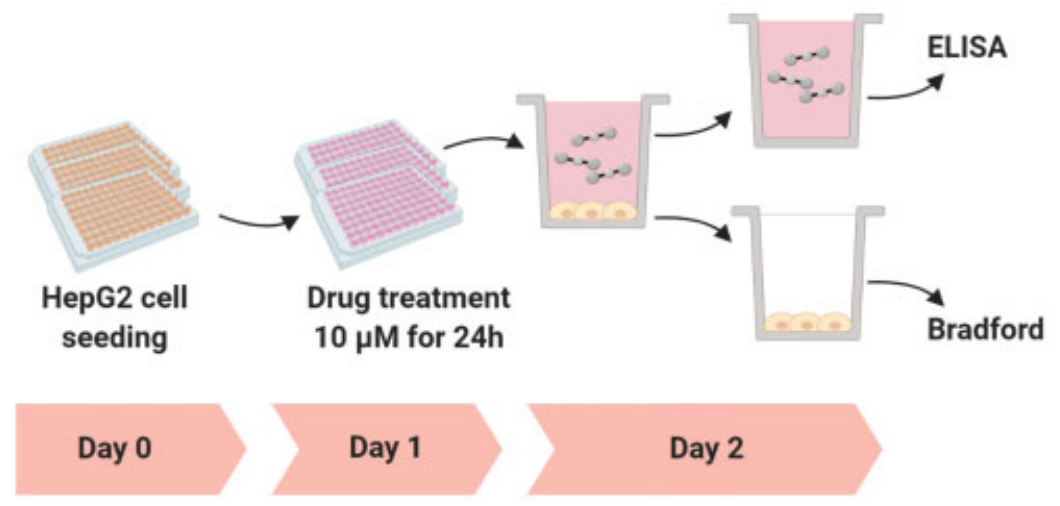

B

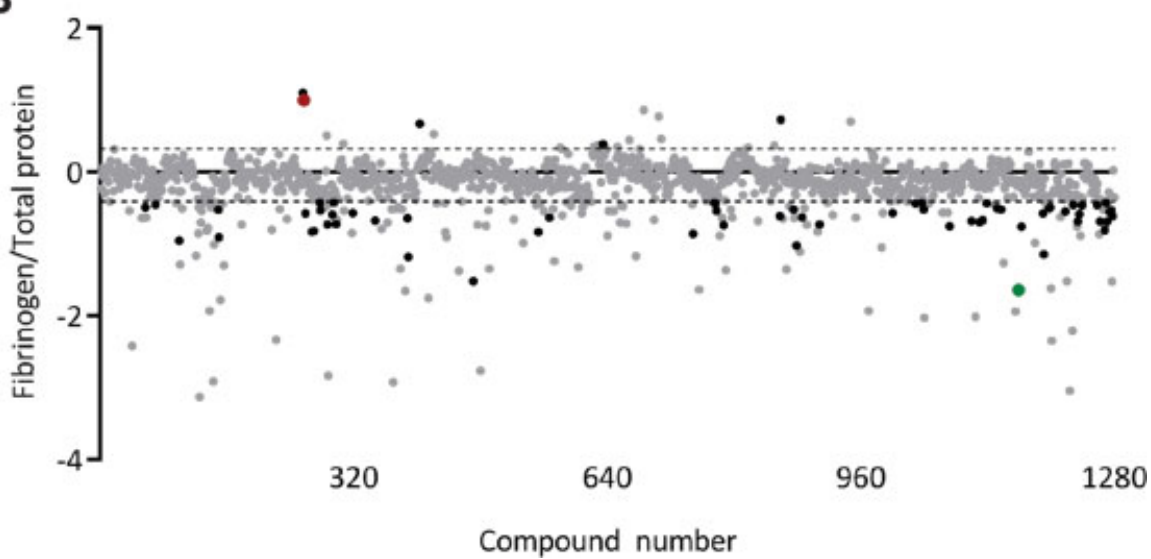

Fig. 1 A chemical screen on fibrinogen production. (A) Schematic representation of the initial chemical screen for fibrinogen production modulators, elaborated with BioRender.com. A total of 1,280 compounds were tested for their effects on fibrinogen production in HepG2 cells. Fibrinogen present in the conditioned medium was measured by ELISA after 24-hour treatments and adjusted to total cellular protein in each well. Compounds were tested in technical triplicates and the screen performed three times. Data were normalized to treatment with DMSO (vehicle) alone. (B) Scatter plot representation of log2 secreted fibrinogen per cellular protein for the 1,280 compounds. The $25 \%$ difference to control cut-off is represented in dotted lines. Black, green, and red dots represent the selected compounds, with the latter representing anthralin and retinoic acid, respectively. ELISA, enzyme-linked immunosorbent assay.

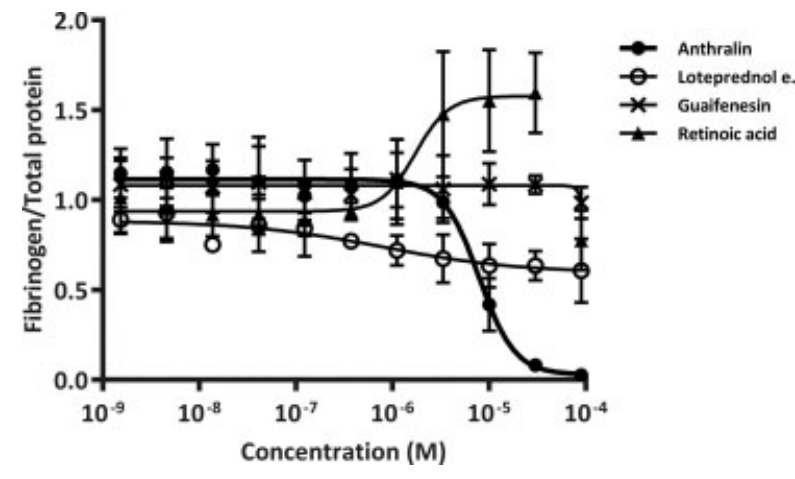

Fig. 2 Dose-response assays for initial screening hits in HepG2 cells. Compounds affecting fibrinogen expression in the described chemical screen were tested at a broad range of concentrations for dose responsiveness and efficacy. Four examples are represented here, with anthralin and retinoic acid giving data that fit sigmoidal response curves, guaifenesin a likely false-positive from the screen, and loteprednol etabonate with weak fibrinogen-lowering activity. Data were normalized to DMSO (vehicle). Assays were made in technical triplicates, and experiments performed three times. Error bars represent standard deviation $(n=3)$. Data for a further 66 molecules from this step in our compound selection are given in

- Supplementary Fig. $\mathbf{S 1}$ (online only). 24-hour incubation from 2dpf to 3dpf (-Fig. 3G). Anthralin and LE reduced fibrinogen production, anthralin significantly, and a small non-significant increase was detected with RA (-Figs. 3H, I). We decided to continue with functional thrombosis testing using anthralin and RA treatments, as anthralin clearly reduced fibrinogen levels in the two treatment regimens, and RA was the only compound increasing fibrinogen in zebrafish larvae. Similar effects of RA were described previously in vitro in HepG2 cells, in primary human hepatocytes and in rats. ${ }^{36}$ Moreover, experiments using antibodies recognizing the fibrinogen $A \alpha$ and $A \alpha E$ chains (see below) showed increased production in 3dpf embryos after a 24-hour incubation with RA. The structures of anthralin and RA are represented in -Supplementary Fig. $\mathbf{S 4}$ (available in the online version). A summary of compounds used at screening steps after the initial screen is given in - Table $\mathbf{1}$.

\section{Thrombocyte Activity and Venous Thrombosis in Zebrafish Larvae}

To study the effects of anthralin and RA on thrombosis in vivo, we used two assays of laser-induced injury of the PCV in 


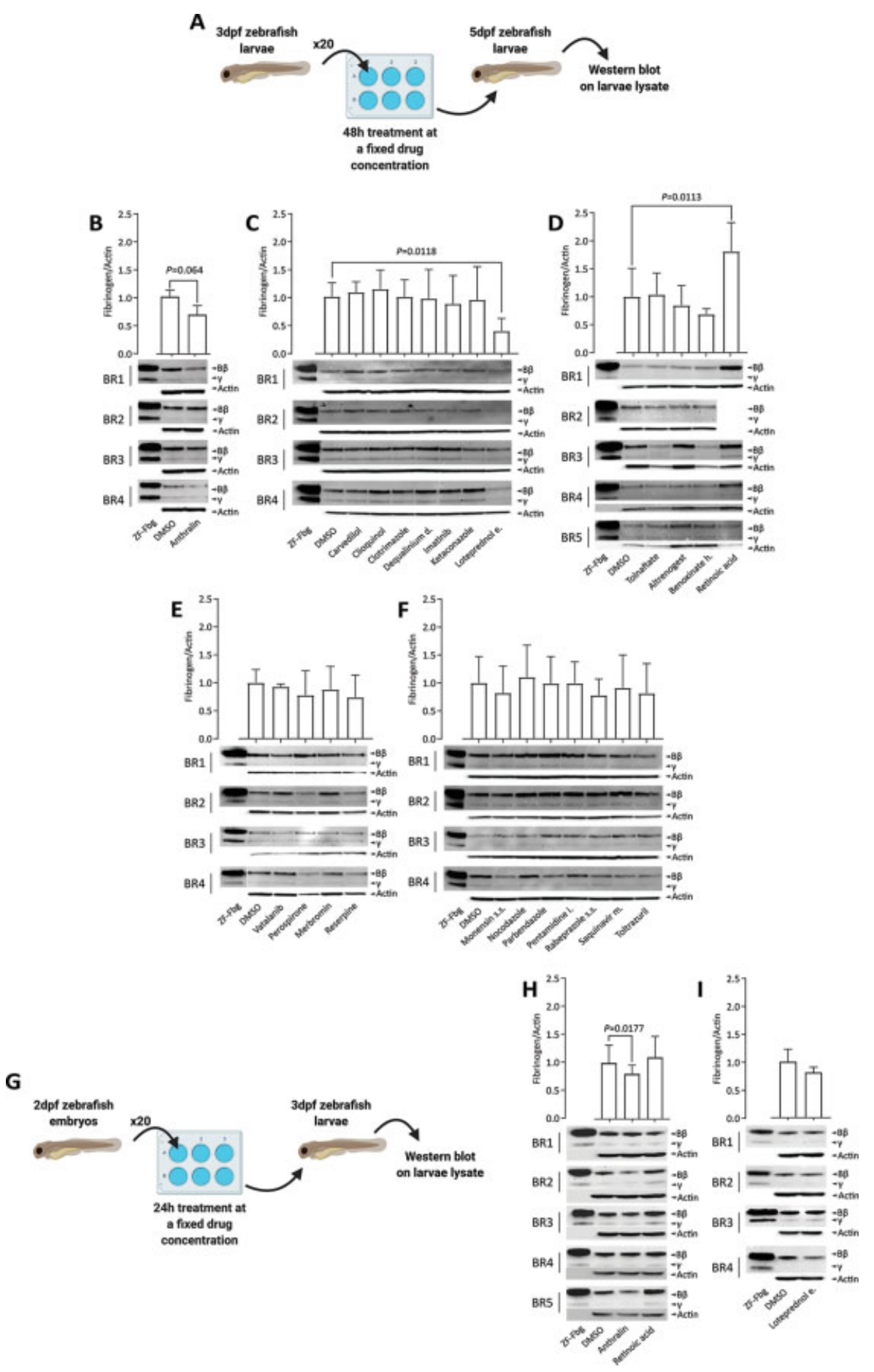

Fig. 3 Fibrinogen production in zebrafish larvae treated with selected compounds. 3dpf Zebrafish larvae were treated for 48 hours with 23 compounds and fibrinogen production was assessed by immunoblotting of the fibrinogen $B \beta$ chain normalized to actin (A-F). Compounds were not all tested at the same time; blots show grouped compounds accordingly. Data from biological replicates (BR) are shown and bar charts represent quantified gel bands. Error bars signify SD ( $n=4$ or 5 ); data were normalized to DMSO. A paired $t$-test $p$-value is shown for certain comparisons. ZF-Fbg is a control sample of recombinant zebrafish fibrinogen produced in mammalian cell culture. The maximum concentrations not revealing toxic effects were determined before the test and constitute the used dosages in these assays $(\mu \mathrm{M})$ : anthralin 0.03; carvedilol 0.33; clioquinol 0.11; clotrimazole 0.037; dequalinium dichloride 0.33; imatinib 9; ketoconazole 1.0; loteprednol etabonate 3.0; tolnaftate 3.0; altrenogest 3.0; benoxinate hydrochloride 3.0; retinoic acid 0.33 ; vatalanib 0.11 ; perospirone 3.0 ; merbromin 0.037 ; reserpine 0.11 ; monensin sodium salt 1.0; nocodazole 0.037 ; parbendazole 0.037 ; pentamidine isethionate 0.33 ; rabeprazole sodium salt 3.0 ; saquinavir mesylate 1.0 ; toltrazuril 1.0. The same analysis was used for anthralin, retinoic acid, and loteprednol etabonate treatment at an earlier developmental time point (3dpf larvae treated for 24 hours only; G-I). Tested concentrations were also verified prior to the tests to exclude toxic developmental effects $(\mu \mathrm{M})$ : Anthralin 0.054; Retinoic acid 0.33; Loteprednol etabonate 3.0. Blot images were cropped and adjusted for brightness and contrast for clarity. In (E), the ZF-fbg control band of BR4 was spliced for consistency with the other blot images. (A) and (G) schematic representations of the two in vivo assays were prepared using BioRender.com. SD, standard deviation. 
Table 1 Selection of compounds during screening steps

\begin{tabular}{|c|c|c|c|c|}
\hline \multirow[t]{3}{*}{ Compound } & \multicolumn{4}{|c|}{ Selection procedures } \\
\hline & A & B & C & $\mathrm{D}$ \\
\hline & $\begin{array}{l}\text { Dose-response } \\
\text { in HepG2 cells }\end{array}$ & $\begin{array}{l}\text { Fibrinogen }>+25 \% \\
\text { or }-25 \% \text { of control in } \\
\text { Huh7 cells }(10 \mu \mathrm{M})\end{array}$ & $\begin{array}{l}\text { Changes in fibrinogen } \\
\text { versus control in } 5 \mathrm{dpf} \\
\text { zebrafish larvae } \\
\text { (48-hour ttt.) }\end{array}$ & $\begin{array}{l}\text { Changes in fibrinogen } \\
\text { versus control in } 3 \mathrm{dpf} \\
\text { zebrafish larvae } \\
\text { (24-hour ttt.) }\end{array}$ \\
\hline Aceclidine hydrochloride & No & NT & NT & NT \\
\hline Acetylsalicylic acid & No & NT & NT & NT \\
\hline Actarit & No & NT & NT & NT \\
\hline Altrenogest & Yes & Yes & No & NT \\
\hline Anthralin & Yes & Yes & Yes $^{\mathrm{a}}$ & Yes \\
\hline Aripiprazole & No & NT & NT & NT \\
\hline Benoxinate hydrochloride & Yes & Yes & No & NT \\
\hline Brimonidine L-tartrate & No & NT & NT & NT \\
\hline Carvedilol & Yes & Yes & No & NT \\
\hline Ceftibuten & No & NT & NT & NT \\
\hline Cinnarizine & No & NT & NT & NT \\
\hline Cisatracurium besylate & Yes & No & NT & NT \\
\hline Clioquinol & Yes & Yes & No & NT \\
\hline Clocortolone pivalate & No & NT & NT & NT \\
\hline Clofilium tosylate & No & NT & NT & NT \\
\hline Clotrimazole & Yes & Yes & No & NT \\
\hline Dequalinium dichloride & Yes & No & NT & NT \\
\hline Dihydroergotamine tartrate & Yes & No & NT & NT \\
\hline Dofetilide & No & NT & NT & NT \\
\hline Doxazosin mesylate & No & NT & NT & NT \\
\hline Ethacrynic acid & No & NT & NT & NT \\
\hline Ethynodiol diacetate & Yes & No & NT & NT \\
\hline Fendiline hydrochloride & No & NT & NT & NT \\
\hline Fenoldopam & Yes & No & No & NT \\
\hline Fipexide hydrochloride & Yes & No & No & NT \\
\hline Fluvastatin sodium salt & Yes & No & No & NT \\
\hline Fosinopril & Yes & No & No & NT \\
\hline Glafenine hydrochloride & Yes & No & No & NT \\
\hline Guaifenesin & No & NT & NT & NT \\
\hline Imatinib & Yes & Yes & No & NT \\
\hline Isotretinoin & Yes & No & NT & NT \\
\hline Itraconazole & Yes & No & NT & NT \\
\hline Ketoconazole & Yes & Yes & No & NT \\
\hline Labetalol hydrochloride & Yes & No & NT & NT \\
\hline Lansoprazole & Yes & No & NT & NT \\
\hline Latanoprost & No & NT & NT & NT \\
\hline Levocabastine hydrochloride & No & NT & NT & NT \\
\hline Loteprednol etabonate & Yes & Yes & Yes & No \\
\hline Lymecycline & Yes & No & NT & NT \\
\hline
\end{tabular}


Table 1 (Continued)

\begin{tabular}{|c|c|c|c|c|}
\hline \multirow[t]{3}{*}{ Compound } & \multicolumn{4}{|c|}{ Selection procedures } \\
\hline & $A$ & B & $C$ & D \\
\hline & $\begin{array}{l}\text { Dose-response } \\
\text { in HepG2 cells }\end{array}$ & $\begin{array}{l}\text { Fibrinogen }>+25 \% \\
\text { or }-25 \% \text { of control in } \\
\text { Huh7 cells }(10 \mu \mathrm{M})\end{array}$ & $\begin{array}{l}\text { Changes in fibrinogen } \\
\text { versus control in 5dpf } \\
\text { zebrafish larvae } \\
\text { (48-hour ttt.) }\end{array}$ & $\begin{array}{l}\text { Changes in fibrinogen } \\
\text { versus control in } 3 \mathrm{dpf} \\
\text { zebrafish larvae } \\
\text { (24-hour ttt.) }\end{array}$ \\
\hline Melengestrol acetate & Yes & No & NT & NT \\
\hline Merbromin & Yes & Yes & No & NT \\
\hline Milnacipran hydrochloride & No & NT & NT & NT \\
\hline Monensin sodium salt & Yes & Yes & No & NT \\
\hline Nocodazole & Yes & Yes & No & NT \\
\hline Oxybutynin chloride & Yes & No & NT & NT \\
\hline Palonosetron hydrochloride & Yes & No & NT & NT \\
\hline Parbendazole & Yes & Yes & No & NT \\
\hline Pentamidine isethionate & Yes & Yes & No & NT \\
\hline Pergolide mesylate & No & NT & NT & NT \\
\hline Perospirone & Yes & Yes & No & NT \\
\hline Proadifen hydrochloride & Yes & No & NT & NT \\
\hline Rabeprazole sodium salt & Yes & Yes & No & NT \\
\hline Reserpine & Yes & Yes $^{b}$ & No & NT \\
\hline Retinoic acid & Yes & Yes & Yes $^{c}$ & Yes \\
\hline Risperidone & Yes & No & NT & NT \\
\hline Saquinavir mesylate & Yes & Yes & No & NT \\
\hline Sildenafil & No & NT & NT & NT \\
\hline Sulfamethazine sodium salt & No & NT & NT & NT \\
\hline Thioguanosine & No & NT & NT & NT \\
\hline Thioproperazine dimesilate & No & NT & NT & NT \\
\hline Thyroxine (L) & No & NT & NT & NT \\
\hline Tolnaftate & Yes & Yes & No & NT \\
\hline Toltrazuril & Yes & Yes & No & NT \\
\hline Valdecoxib & Yes & No & NT & NT \\
\hline Vatalanib & Yes & Yes & No & NT \\
\hline Zalcitabine & No & NT & NT & NT \\
\hline
\end{tabular}

Abbreviations: NT not tested, ttt., treatment.

Note: List of compounds selected after the initial screening assay and decision to test in subsequent experiments by selection procedures.

Compounds initially found to increase or decrease fibrinogen levels are in red or green, respectively. Selection procedures A and B were based on changes in fibrinogen levels measured by ELISA of cell-conditioned medium normalized to cellular protein and vehicle (DMSO). C and D were based on changes in fibrinogen antigen in zebrafish larvae, normalized to $\beta$-actin levels and a control condition (DMSO), measured by immunoblotting.

a Selected for further study as the result was close to significant $(p=0.064)$.

bIncluded for the next screening step as the fibrinogen change obtained with $10 \mu \mathrm{M}$ was close to the $25 \%$ selection cut-off (23\%) and statistically significant (paired $t$-test).

'Selected for additional experiments as the only compound leading to increased fibrinogen levels in zebrafish larvae.

zebrafish larvae. The first test measured thrombocyte adhesion and accumulation at the injury site in $5 \mathrm{dpf} \operatorname{Tg}$ (itga2b: EGFP) larvae with fluorescent thrombocytes. Larvae were treated for 48 hours with anthralin, RA, or vehicle (DMSO), from 3 to $5 \mathrm{dpf}$, and fluorescence accumulation monitored over time after injury. Two parameters were measured: the thrombus area expressed as the percentage of pixels reach- ing a defined fluorescence threshold within a precise area, and a comparison of the quantity of thrombocytes present at the injury site by averaging the fluorescence intensity of pixels around the wound.

Thrombocyte adhesion-aggregation dynamics were affected by anthralin and RA in a manner that correlated with fibrinogen levels. Fibrinogen-lowering anthralin treatment 
led to aggregates with lower mean fluorescence levels and a trend toward a smaller size ( - Figs. 4A, B). When comparing individual time points post-laser, an unpaired $t$-test detected differences $(p<0.05)$ between mean fluorescence values ( - Fig. 4B), but not in areas ( - Fig. 4A), for anthralin versus DMSO in time lapse frames 610 to 817 and 988 to 1108 . A higher mean fluorescence intensity at the injury site was obtained with RA treatment, but this was not statistically significant, while displaying a similar area (-Figs. 4C, D). This suggests that RA treatment may favor thrombocyte aggregation while anthralin behaves as an antithrombotic entity.

To exclude effects of RA or anthralin on thrombocyte development, which may influence their activity in the laser-injury assay, we used fluorescence-activated cell sorting to assess thrombocyte numbers and fluorescence intensity in cell suspensions from larvae with and without treatments. The percentage of GFP-positive cells and mean relative fluorescence intensity were measured. We also analyzed two GFP-positive subsets, GFPhigh corresponding to more mature thrombocytes, and $\mathrm{GFP}^{\mathrm{low}}$ which are precursors expressing the itga $2 b$ promoter-driven transgene. ${ }^{37}$ Minor or no differences were measured in these parameters after anthralin treatment (-Supplementary Fig. S5 [avail- able in the online version]) suggesting the effects of this compound in the laser-induced thrombocyte aggregation assays cannot be explained by lower thrombocyte numbers or their relative fluorescence after treatments. However, RA treatment gave a trend toward an increased number of GFPpositive cells. This indicates that any enhancement of thrombocyte aggregation dynamics upon treatment with this compound may not be exclusively fibrinogen-dependent.

We measured the time to occlusion (TTO) after laser injury of the PCV in 3dpf larvae. Laser-induced venous thrombosis is fibrinogen-dependent, as fibrinogen-deficient larvae largely fail to support occlusive thrombosis. ${ }^{20}$ The TTO in anthralin- and RA-treated larvae correlated with changes in fibrinogen (-Fig. 5). Anthralin prolonged the mean TTO (27.4 seconds, standard deviation [SD]: 10.0, $n=59$ ) and RA shortened it ( 15.3 seconds, SD: $4.2, n=60$ ), compared with controls (DMSO, 23.8 seconds, SD: 6.9, $n=61$ ). The difference between anthralin and control treatment remains significant after removal of the outlier in the anthralin group (mean: $26.6 \mathrm{sec}$, SD: 7.9, $p=0.0391$ ).

To exclude changes in the morphology of fibrinogenexpressing tissues-the yolk syncytial layer and the liveras a source of altered fibrinogen levels in anthralin- or RAtreated larvae, we used whole-mount in situ hybridization to
A

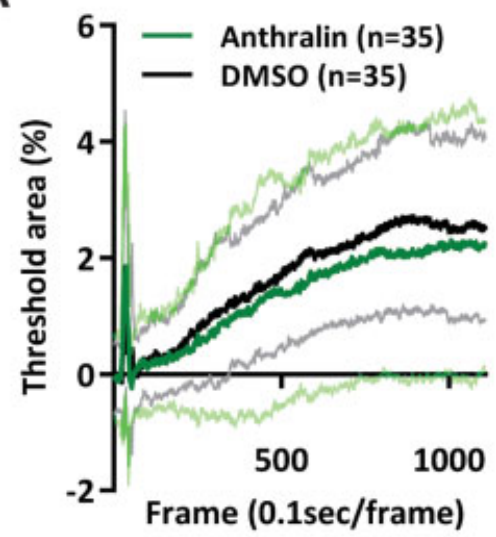

C

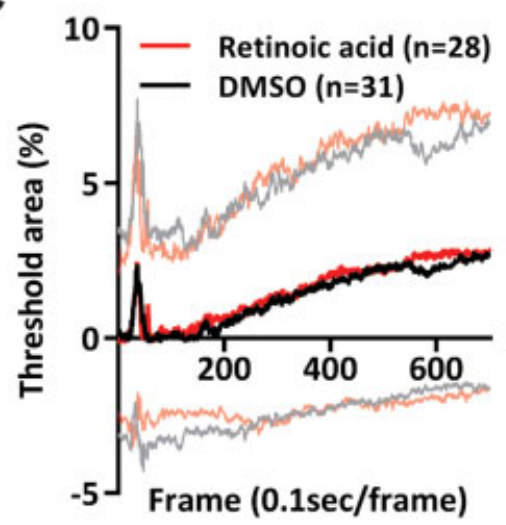

B

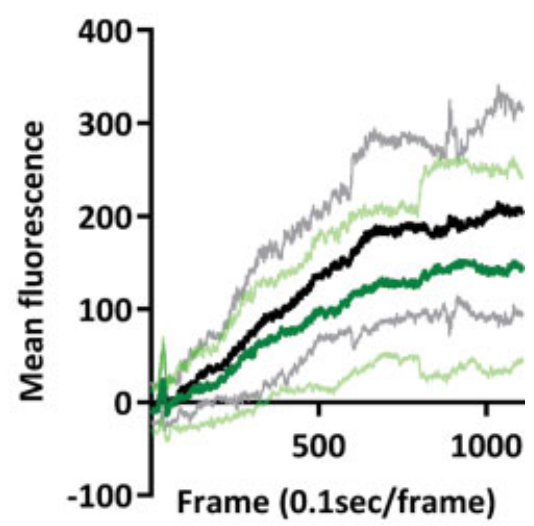

D

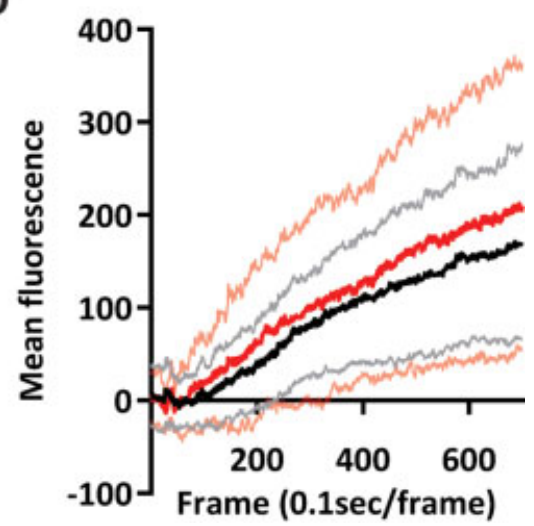

Fig. 4 Laser-induced thrombocyte aggregation. Hemizygous $5 \mathrm{dpf} \mathrm{Tg}$ (itga2b-EGFP) zebrafish larvae were treated for 48 hours with anthralin (A, B) or retinoic acid (C, D) or vehicle alone (DMSO), and subjected to laser injury in the PCV. Accumulation of fluorescent thrombocytes around the injury site was recorded and quantified. Two parameters were analyzed, the thrombus area determined from the percentage of pixels that reach a threshold fluorescence intensity (A, C) and the number of thrombocytes present in the clot (B, D) inferred by the mean fluorescence intensity. Tested concentrations: Anthralin $0.03 \mu \mathrm{M}$; Retinoic acid $0.33 \mu \mathrm{M}$. Mean and SD are represented by darker and lighter lines, respectively $(n=28-$ 35). PCV, posterior cardinal vein; SD, standard deviation. 
A
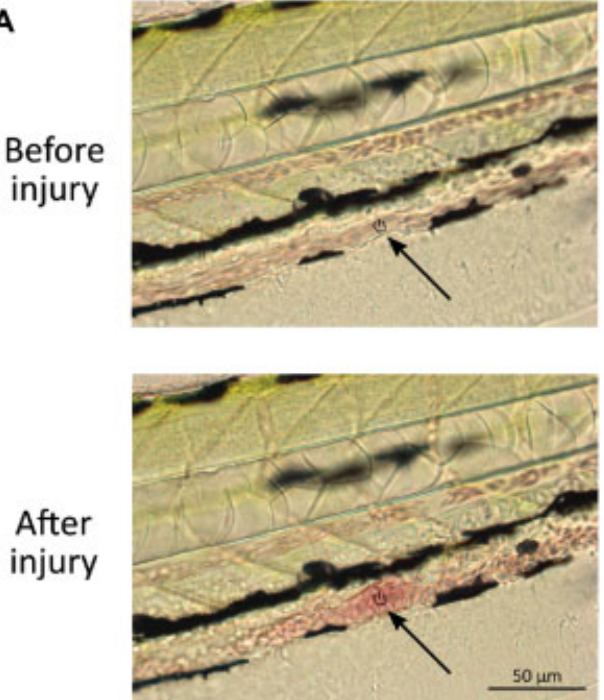

B

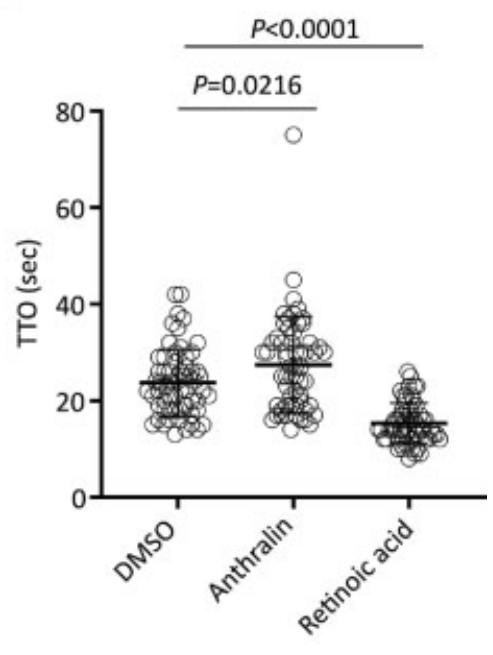

Fig. 5 Laser-induced venous thrombosis. In (A), a typical laser-induced occlusive thrombotic event is shown in images before and after injury of the $3 \mathrm{dpf}$ zebrafish posterior cardinal vein. The arrow points to the injury site. In (B), the time to occlusion (TTO) is shown after vehicle (DMSO), anthralin, or retinoic acid treatment. Individual larvae are represented by open circles. Tested concentrations: Anthralin $0.054 \mu \mathrm{M}$; Retinoic acid $0.33 \mu \mathrm{M}$. $p$-values from unpaired $t$-tests are presented, $n=61,59$, and 60 for DMSO-, anthralin-, and retinoic acid-treated larvae, respectively. Mean and standard deviation are embedded in the dot plots.

probe the fga mRNA in 3dpf larvae (-Supplementary Fig. $\mathbf{S 6}$ [available in the online version]). The yolk syncytial layer and liver primordium structures and fga expression could be seen with all treatments, as described previously in control larvae. ${ }^{28}$ Treatment with RA leads to stronger in situ hybridization staining, suggesting a RA-induced increased steadystate fga mRNA.

\section{Consequences of Moderate Variations in Fibrinogen Levels on Experimental Venous Thrombosis}

Since RA affects fibrinogen production by increasing its expression, which we measured in both HepG2 cells and zebrafish larvae (-Figs. 6A, B) and was reported previous$\mathrm{ly},{ }^{36}$ we treated fibrinogen deficient $\left(\mathrm{fga}^{-1-}\right)^{19}$ and morpholino-injected embryos with RA. This was to provide additional evidence as to whether the effects of RA on venous thrombosis (shortened TTO) were fibrinogen-dependent.

We confirmed that fibrinogen-deficient 3dpf zebrafish larvae do not form occlusive venous thrombi after laser injury ( $f g a^{-1-}+$ DMSO alone, $n=10$ ), as reported previously, ${ }^{20}$ and observed that treatment with RA had no effect on this inability to support venous occlusion $\left(f g a^{-1-}+R A\right.$, no occlusion measured, $n=13$ ). Therefore, RA cannot promote venous thrombosis in the absence of fibrinogen.

With the morpholino approach we could then induce moderate lowering of fibrinogen expression, assess its effect on 3dpf laser-induced TTO, and test whether RA consistently affects the TTO at different fibrinogen levels. Injection of different quantities of a fibrinogen morpholino into early zebrafish embryos to knock down fga mRNA gave a dosedependent decrease in fibrinogen (-Figs. 6C-F). This correlated with results obtained in the thrombosis assay: increasing amounts of morpholino resulted in a dose-dependent decrease in the percentage of laser-induced occlusive inju- ries and increased the TTO in those that did occlude (-Figs. 6G, H). Treatment with RA, in any of the morpholino settings, increased fibrinogen mRNA expression and protein level, increased the percentage of occlusive thrombi after laser injury and shortened the TTO in the occlusive wounds. Prior to this, we tested the specificity and excluded toxic effects of the morpholino by comparing its activity to a control morpholino at $6 \mathrm{ng}$ and $2 \mathrm{ng}$ per embryo (-Supplementary Fig. $\mathbf{5 7}$ [available in the online version]). Our data support the inverse correlation between fibrinogen level and laser-induced TTO, and that the shortening of TTO with RA could be, at least in part, mediated by its effect on fibrinogen production.

\section{Mechanism-of-Action of Anthralin on Fibrinogen Production}

To assess whether anthralin affected fibrinogen levels at the mRNA level, we isolated RNA and used RT-qPCR to detect fibrinogen cDNA in both HepG2 cells and 5dpf zebrafish larvae with and without anthralin treatment. While anthralin significantly reduced fibrinogen mRNA levels in HepG2 cells, in larvae it did not ( - Figs. 7A, B). This could have been due to the lower nontoxic tolerated anthralin concentration used in vivo. To generate hypotheses concerning the possible mechanism-of-action of anthralin on fibrinogen beyond effects on the steady-state fibrinogen mRNA level, we used a proteomic approach to look for changes in the overall protein component of lysates from HepG2 cells and zebrafish larvae after incubation with anthralin. Two independent experiments were made for each. Proteins differentially represented after anthralin treatment compared with controls were listed for each replicate after Fisher's exact tests using Scaffold software (see the Methods section). We aimed to select proteins less represented after anthralin treatment 

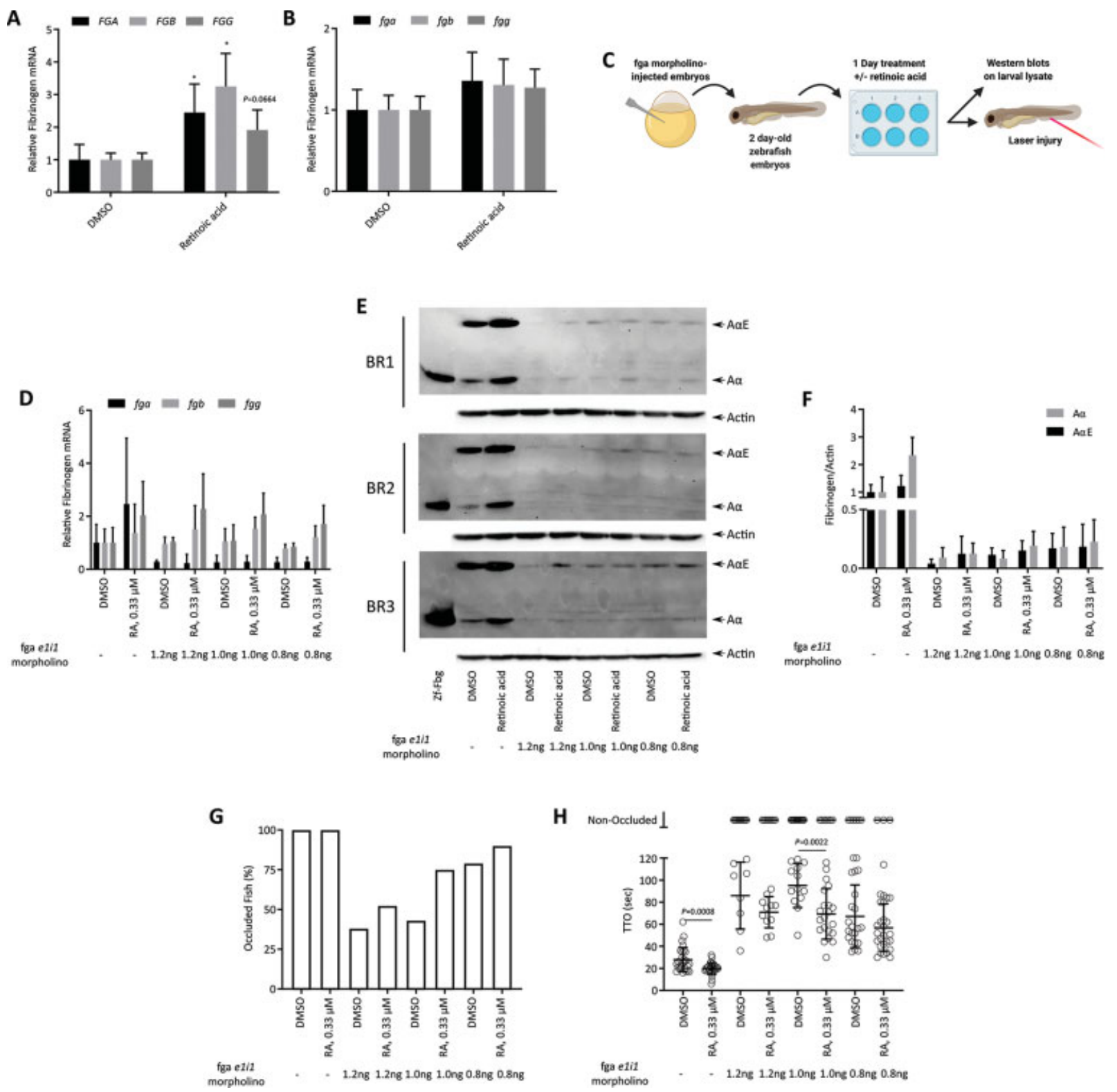

Fig. 6 Fibrinogen modulation directly correlates with venous thrombosis in zebrafish larvae. mRNA of the three fibrinogen chains from HepG2 cells (A) and 5dpf zebrafish embryos (B) were quantified by RT-qPCR. Results for paired $t$-tests are presented. The symbol $\left({ }^{*}\right)$ indicates $p$ value $<0.05, n=3$; error bars represent SD. The tested concentrations of retinoic acid were 0.123 and $0.33 \mu \mathrm{M}$ in (A) and (B), respectively. Fibrinogen knock-down with a morpholino was used to demonstrate the relationship between fibrinogen levels and venous thrombosis with retinoic acid treatment. The experiment is shown schematically in (C), generated with graphics from BioRender.com. In (D), the effects of 1.2, 1.0 , or $0.8 \mathrm{ng} /$ embryo of fibrinogen morpholino, with or without retinoic acid treatment, on zebrafish fibrinogen mRNA $(n=3)$ are shown. Error bars represent SD. The effects of 1.2, 1.0, or $0.8 \mathrm{ng} /$ embryo of fibrinogen morpholino on fibrinogen protein levels, in the presence or absence of retinoic acid, were assessed by immunoblotting analyses using an antibody recognizing A $\alpha$ and $A \alpha E$ chains and normalization to actin. Blots from three biological replicate (BR1-3) experiments are shown in (E), with the respective quantification displayed in (F). Error bars represent SD, $n=3$. Blot images were cropped and adjusted for brightness and contrast for clarity. The percentages of larvae with occluding venous thrombosis after laser injury are displayed in $(\mathbf{G})$, with the times to occlusion presented in $(\mathbf{H})$. Mean and SD are embedded in the dot plots. $p$-Values are from unpaired $t$-tests, which only took into account occlusive thrombi $(n=13-30)$. SD, standard deviation.

in both of the HepG2 cell or zebrafish replicates for biological pathway enrichment analysis using DAVID (https://david. ncifcrf.gov/), looking for pathways or protein domains that were differentially detected (see - Supplementary Data [available in the online version]).

Data obtained using proteins less represented in HepG2 cell anthralin-treated samples $(p \leq 0.05)$ suggest a general effect of the compound on proteins destined for the canonical secretory pathway. Among the enriched pathways were platelet degranulation, secreted, extracellular space, extracellular region, and extracellular exosome ( - Fig. 7C). Only two proteins were less represented $(p \leq 0.05)$ in larval zebrafish samples from replicate analyses, preventing effective analysis with DAVID. Nevertheless, we were interested to know whether any similarities to the HepG2 cell results could be suggested by the zebrafish proteins that were underrepresented after anthralin 
A

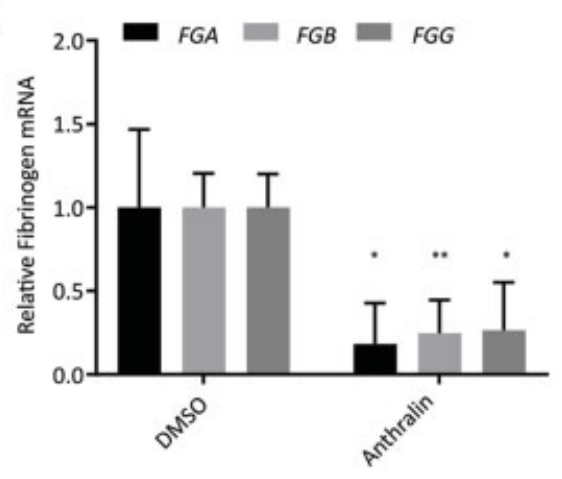

B

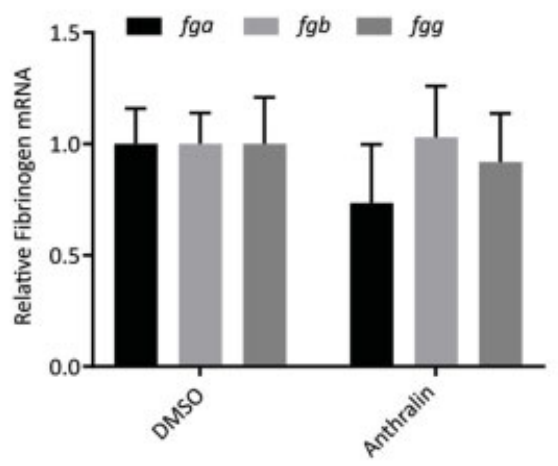

C

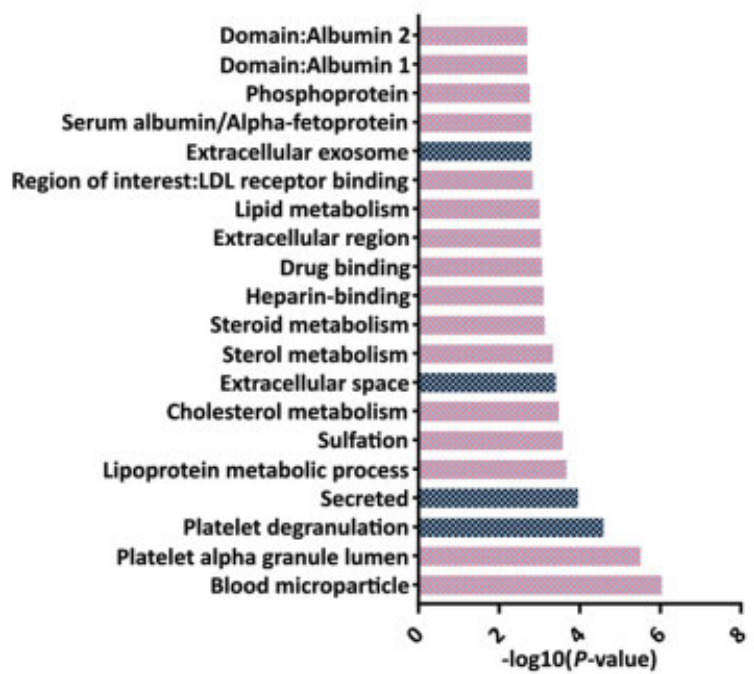

D

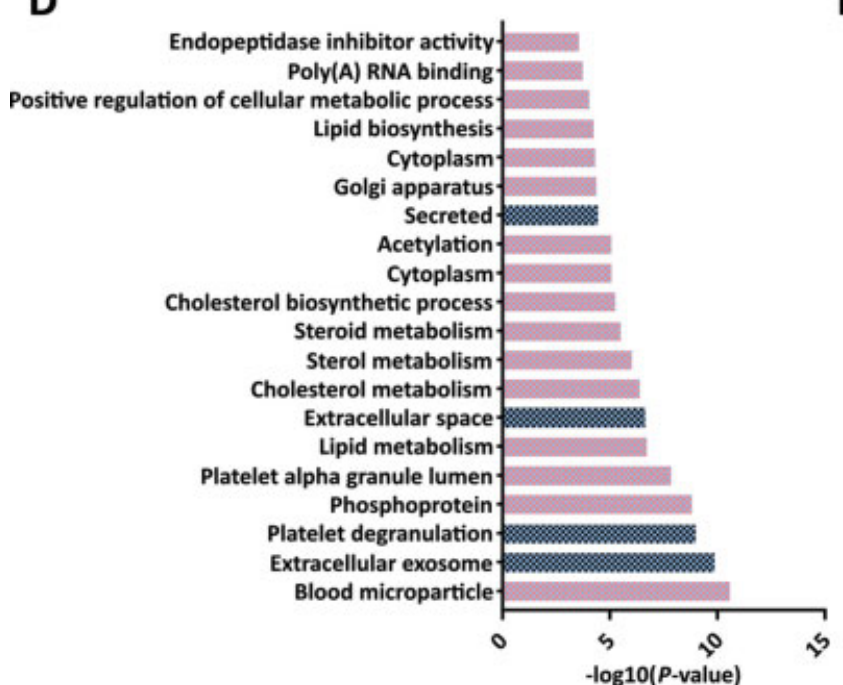

$\mathbf{E}$

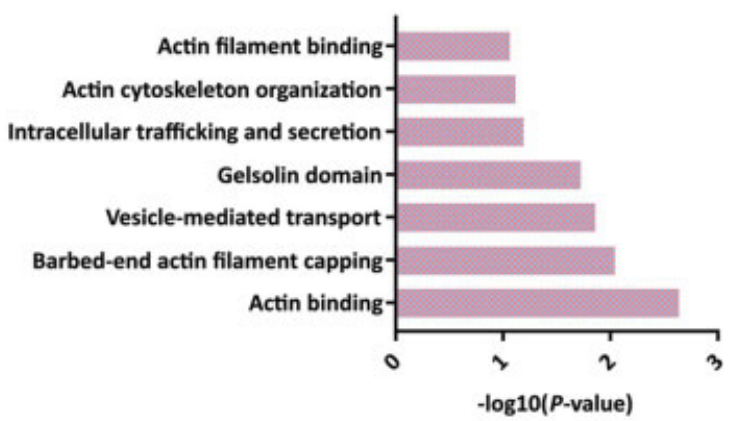

Fig. 7 Effects of anthralin on fibrinogen expression and the proteome of HepG2 cells and zebrafish larvae. mRNA levels of the three fibrinogen chains from HepG2 cells (A) and 5dpf zebrafish larvae (B) after treatment with anthralin were measured by RT-qPCR. Results for paired $t$-tests are presented; $\left({ }^{*}\right)$ for $p$-value $<0.05 ;\left({ }^{* *}\right)$ for $p$-value $<0.001$; error bars represent $S D, n=3$. Enrichment analysis using proteins consistently less represented in two biological replicates of the proteomes of HepG2 cells ( $C$ and $D)$ or $5 \mathrm{dpf}$ zebrafish larvae (E) treated with anthralin (C: $p \leq 0.05$; $D$ and $E: p \leq 0.31)$. The outcome of the chart report tool from DAVID is presented with the top 20 pathways or domains shown for the HepG2 cell analyses (C, D) and the complete list for zebrafish larvae (E). This is an annotation-term-focused view and lists terms from common bioinformatic resources, including the three sub-ontologies of GO term (cellular component, biological process, or molecular function), UniProt keywords, Uniprot Sequence Feature, InterPro, and Clusters of Orthologous Groups. Groupings related to the secretory pathway found in both analyses performed with HepG2 samples are highlighted in blue in (C) and (D). 


\section{Chemical modulators of fibrinogen production and their impact on venous thrombosis}
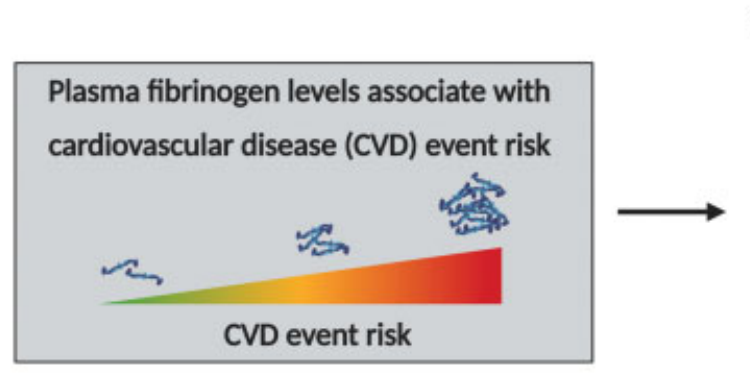

1280 drugs screened in HepG2 cells
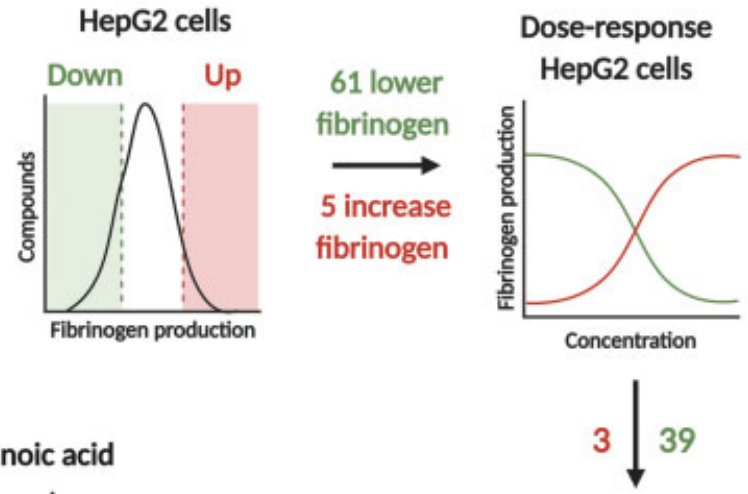

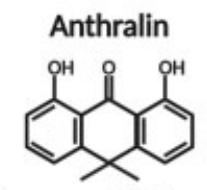

Decreased fibrinogen Antithrombotic

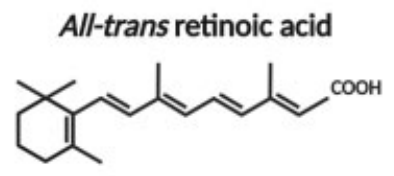

Increased fibrinogen Prothrombotic
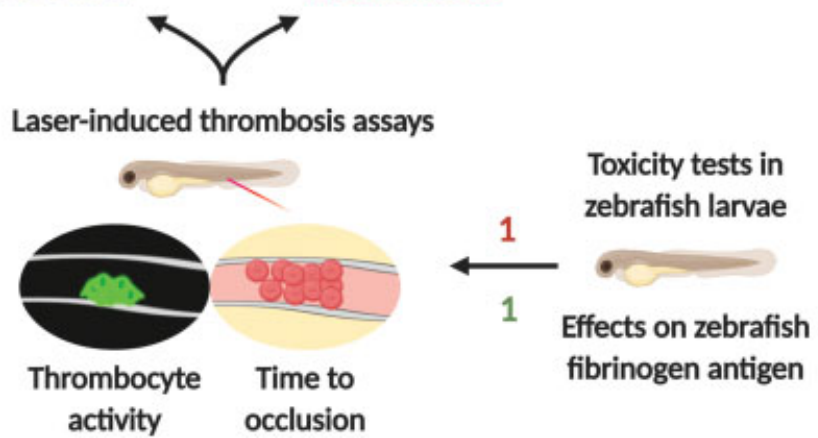

Fig. 8 Visual Summary: Plasma fibrinogen concentration correlates with CVD event risk and high fibrinogen levels are considered prothrombotic. A chemical library of bioactive compounds was screened for altering fibrinogen production. Active compounds were identified by a broad screen in HepG2 cells and then by a series of selection steps in cellular models and zebrafish larvae. Anthralin treatment led to lower fibrinogen production, whereas all-trans retinoic acid increased it. Using laser-induced venous thrombosis assays in zebrafish larvae, anthralin showed antithrombotic activity whereas all-trans retinoic acid was prothrombotic (created with BioRender.com).

treatment. By setting a Fisher's exact test $p$-value cut-off to 0.31 , greatly reducing the likely significance of the analysis outcome, similar results were seen in HepG2 cells to those at $p$ $\leq 0.05$ ( - Fig. 7D). In data from zebrafish samples, the vesiclemediated transport and intracellular trafficking and secretion were found within the few enrichment terms detected with the nonsignificant analysis $p$-value (-Fig. 7E).

We compared our proteome data with a listing of the proteins in the HepG 2 cell secretome. ${ }^{38}$ of the secreted proteins detected in our experiments with HepG2 cells, peptides from $>80 \%$ of those proteins with a signal peptide were less abundant after anthralin treatment. For proteins without a signal peptide, the percentage with lower abundance following anthralin treatment was 19 and 32\% for the two experimental replicates, further linking the effect of anthralin to canonical secretion.

\section{Discussion}

In this study a chemical screen was used to identify molecules which alter fibrinogen protein production in human cell lines and zebrafish larvae and affect experimental venous throm- bosis. Our data revealed anthralin as a drug that consistently leads to lower fibrinogen in the settings tested, prolonged venous thrombosis time, and affected thrombocyte activity. RA, previously known to increase plasma fibrinogen in rats, ${ }^{36}$ was identified as a fibrinogen-increasing agent with opposite effects to anthralin in the TTO assay and displayed a trend toward enhanced thrombocyte aggregation compared with control. The detection of RA in this context confirmed the ability of our screening method to uncover molecules with reproducible effects on fibrinogen levels. These results, together with data from experiments with a fibrinogen-targeting morpholino in which larval fibrinogen levels correlate with laser-induced thrombosis times, add to previous findings in a hyperfibrinogenemic mouse model, ${ }^{17}$ indicating high fibrinogen as a mediator of a prothrombotic environment. A visual summary of this study is provided (-Fig. 8).

Many acquired or inherited factors can contribute to thrombosis. The acquired factors include medicines, hence the need to assess medication when evaluating thrombosis risk. RA is used topically to treat acne, and for acute promyelocytic leukemia (APL). There is a possible link between our data and clinical use of RA. Some APL patients were reported to have low 
plasma fibrinogen ( $<0.1 \mathrm{mg} / \mathrm{mL}$ in $61 \%$ of patients, $n=34$ ) and suffer from hemorrhages. ${ }^{39}$ In contrast, treatment with RA has been shown to give a rapid correction of fibrinogen levels and increase thrombotic events. ${ }^{40-42}$ Apart from the plausible contribution of increased fibrinogen production, another potential source for this prothrombotic effect is thrombocytosis seen in RA-treated APL patients. ${ }^{43}$ Here we also show that RA treatment increased thrombocyte cell fraction in a nonstatistically significant manner. Effects of RA on platelet activity have also been reported. RA reduced platelet activation in dietinduced atherosclerosis in rabbits, ${ }^{44}$ and inhibited platelet aggregation, spreading, dense-granule secretion, and clot retraction in vitro. ${ }^{45}$ While we cannot exclude a contribution of the increased number of thrombocytes, possible direct effects of RA include thrombocyte responses to injury, or even an increased production of other key coagulation factors through the same mechanism as behind increased fibrinogen production (mediated by RA and the retinoid X nuclear receptors ${ }^{36}$ ), our data showed a trend toward increased thrombocyte binding after RA treatment that correlated with increased fibrinogen.

Anthralin was identified as a compound giving antithrombotic effects in our study. It is used in the topical treatment of psoriasis, ${ }^{46}$ but its molecular target remains elusive. Psoriasis is associated with thrombosis that may result from increased levels of coagulation factors, including fibrinogen, ${ }^{47}$ and a psoriasis-related genetic locus is associated with plasma fibrinogen levels in women. ${ }^{48}$ These links may be due to the inflammatory nature of psoriasis and sensitivity of fibrinogen production to inflammatory stimuli. General anticoagulant activity has been described previously for anthralin. ${ }^{49}$ Mononuclear leukocytes from psoriatic patients presented enhanced cell-bound procoagulant activity when compared with cells from healthy patients, a phenomenon that was normalized upon treatment with anthralin.

Our preliminary proteomic studies on the mechanism of fibrinogen lowering with anthralin in cultured cells and zebrafish larvae support the hypothesis that it is driven by an effect on the canonical secretory pathway. Impaired fibrinogen secretion has been reported with HepG2 cells treated with Brefeldin $A,{ }^{50}$ a chemical known to block protein export. Nevertheless, this hypothesis, for which validation requires further experimental support, implies that anthralin can have global effects on secreted proteins and would show poor selectivity for fibrinogen lowering in vivo. However, the outcome of a general effect on secretion would presumably depend on the physiological functional threshold concentration for each secreted protein affected. If dosedependent, the effects of anthralin on thrombosis might therefore be fine-tuned by the dosage to optimize the specificity of its functional impact.

In the search for venous thrombosis modulators, drug repurposing offers the advantage of a potentially faster introduction of lead compounds into clinical evaluation, since many of the drug development stages have already been performed. ${ }^{51}$ However, the use of anthralin for venous thrombosis prevention, in addition to the aforementioned potential pleiotropic effects on protein secretion, faces another important current limitation because it is adminis- tered as a topical skin treatment and would therefore require toxicology testing and perhaps further development to be envisaged as a systemic therapy.

In summary, our study found small molecules capable of modulating fibrinogen production in vitro and in vivo. Using functional assays of venous thrombosis that are sensitive to changes in fibrinogen levels, we show that treatment with these compounds leads to predictable changes in occlusive venous thrombosis and thrombocyte aggregation. This reinforces the concept that fibrinogen levels can directly influence venous thrombosis and the potential benefit of a fibrinogen-lowering therapy for prevention of the latter. While the usefulness of anthralin in this regard could be limited given its potential broader effects on protein secretion and current formulation as an ointment, the prothrombotic effect of RA treatment may have clinical consequences.

\section{What is known about this topic?}

- High plasma fibrinogen levels associate with increased cardiovascular disease (CVD) event risk.

- Increased plasma fibrinogen leads to a thrombophilic state in a mouse model.

\section{What does this paper add?}

- A screen of bioactive compounds identified small molecules that modulate fibrinogen production in vitro and in vivo: anthralin decreases fibrinogen, retinoic acid increases it.

- Changes in fibrinogen production correlate with laserinduced thrombosis in larval zebrafish assays.

- Retinoic acid is prothrombotic while anthralin shows antithrombotic activity and could be an entry point to a novel antithrombotic strategy.

\section{Funding}

This study was supported by a Swiss National Science Foundation grant (\#31003A_152633) to M. NeermanArbez and a Novartis Foundation for Medical-Biological Research grant to R.J. Fish. We thank the University of Geneva Faculty of Medicine Proteomics, Flow Cytometry and Bioimaging services for their technical assistance and advice.

\section{Conflict of Interest}

R. J. Fish has received travel support from Novo Nordisk. All other authors have no conflict of interest to declare.

\section{References}

1 Raskob GE, Angchaisuksiri P, Blanco AN, et al; ISTH Steering Committee for World Thrombosis Day. Thrombosis: a major contributor to global disease burden. Arterioscler Thromb Vasc Biol 2014;34(11):2363-2371 
2 Wendelboe AM, McCumber M, Hylek EM, Buller H, Weitz JI, Raskob GISTH Steering Committee for World Thrombosis Day. Global public awareness of venous thromboembolism. J Thromb Haemost 2015;13(08):1365-1371

3 Lozano R, Naghavi M, Foreman K, et al. Global and regional mortality from 235 causes of death for 20 age groups in 1990 and 2010: a systematic analysis for the Global Burden of Disease Study 2010. Lancet 2012;380(9859):2095-2128

4 Coleman CI, Sobieraj DM, Winkler S, et al. Effect of pharmacological therapies for stroke prevention on major gastrointestinal bleeding in patients with atrial fibrillation. Int J Clin Pract 2012;66(01):53-63

5 Ahrens I, Lip GY, Peter K. New oral anticoagulant drugs in cardiovascular disease. Thromb Haemost 2010;104(01):49-60

6 Ansell J, Hirsh J, Hylek E, Jacobson A, Crowther M, Palareti G. Pharmacology and management of the vitamin $\mathrm{K}$ antagonists: American College of Chest Physicians Evidence-Based Clinical Practice Guidelines (8th Edition). Chest 2008;133(6, Suppl):160S-198S

7 Berger JS, Krantz MJ, Kittelson JM, Hiatt WR. Aspirin for the prevention of cardiovascular events in patients with peripheral artery disease: a meta-analysis of randomized trials. JAMA 2009; 301(18):1909-1919

8 Ageno W, Gallus AS, Wittkowsky A, Crowther M, Hylek EM, Palareti G. Oral anticoagulant therapy: Antithrombotic Therapy and Prevention of Thrombosis, 9th ed: American College of Chest Physicians Evidence-Based Clinical Practice Guidelines. Chest 2012;141(2, Suppl):e44S-e88S

9 Weitz JI, Eikelboom JW, Samama MM. New antithrombotic drugs: Antithrombotic Therapy and Prevention of Thrombosis, 9th ed: American College of Chest Physicians Evidence-Based Clinical Practice Guidelines. Chest 2012;141(2, Suppl):e120S-e151S

10 Jagadeeswaran P, Cooley BC, Gross PL, Mackman N. Animal models of thrombosis from zebrafish to nonhuman primates: use in the elucidation of new pathologic pathways and the development of antithrombotic drugs. Circ Res 2016;118(09):1363-1379

11 Weisel JW, Litvinov RI. Fibrin formation, structure and properties. Subcell Biochem 2017;82:405-456

12 Mosesson MW, Siebenlist KR, Meh DA. The structure and biological features of fibrinogen and fibrin. Ann N Y Acad Sci 2001; 936:11-30

13 Kant JA, Fornace AJ Jr, Saxe D, Simon MI, McBride OW, Crabtree GR. Evolution and organization of the fibrinogen locus on chromosome 4: gene duplication accompanied by transposition and inversion. Proc Natl Acad Sci U S A 1985;82(08):2344-2348

14 Espitia Jaimes C, Fish RJ, Neerman-Arbez M. Local chromatin interactions contribute to expression of the fibrinogen gene cluster. J Thromb Haemost 2018;16(10):2070-2082

15 Danesh J, Lewington S, Thompson SG, et al; Fibrinogen Studies Collaboration. Plasma fibrinogen level and the risk of major cardiovascular diseases and nonvascular mortality: an individual participant meta-analysis. JAMA 2005;294(14):1799-1809

16 Zhang Y, Zhu C-G, Guo Y-L, et al. Higher fibrinogen level is independently linked with the presence and severity of newonset coronary atherosclerosis among Han Chinese population. PLoS One 2014;9(11):e113460

17 Machlus KR, Cardenas JC, Church FC, Wolberg AS. Causal relationship between hyperfibrinogenemia, thrombosis, and resistance to thrombolysis in mice. Blood 2011;117(18):4953-4963

18 Suh TT, Holmbäck K, Jensen NJ, et al. Resolution of spontaneous bleeding events but failure of pregnancy in fibrinogen-deficient mice. Genes Dev 1995;9(16):2020-2033

19 Fish RJ, Di Sanza C, Neerman-Arbez M. Targeted mutation of zebrafish fga models human congenital afibrinogenemia. Blood 2014;123(14):2278-2281

$20 \mathrm{Hu}$ Z, Lavik KI, Liu Y, et al. Loss of fibrinogen in zebrafish results in an asymptomatic embryonic hemostatic defect and synthetic lethality with thrombocytopenia. J Thromb Haemost 2019;17 (04):607-617
21 Vilar R, Fish RJ, Casini A, Neerman-Arbez M. Fibrin(ogen) in human disease: both friend and foe. Haematologica 2020;105 (02):284-296

22 Perisanidis C, Psyrri A, Cohen EE, et al. Prognostic role of pretreatment plasma fibrinogen in patients with solid tumors: a systematic review and meta-analysis. Cancer Treat Rev 2015;41 (10):960-970

23 Berger MD, Heini AD, Seipel K, Mueller B, Angelillo-Scherrer A, Pabst $T$. Increased fibrinogen levels at diagnosis are associated with adverse outcome in patients with acute myeloid leukemia. Hematol Oncol 2017;35(04):789-796

24 Pedersen A, Stanne TM, Redfors P, et al. Fibrinogen concentrations predict long-term cognitive outcome in young ischemic stroke patients. Res Pract Thromb Haemost 2018;2(02):339-346

25 Zhang Y, Zhang X, Liu D, et al. Elevated fibrinogen levels in neuromyelitis optica is associated with severity of disease. Neurol Sci 2016;37(11):1823-1829

26 Fort A, Borel C, Migliavacca E, Antonarakis SE, Fish RJ, NeermanArbez M. Regulation of fibrinogen production by microRNAs. Blood 2010;116(14):2608-2615

27 Keserü GM, Makara GM. The influence of lead discovery strategies on the properties of drug candidates. Nat Rev Drug Discov 2009;8 (03):203-212

28 Fish RJ, Vorjohann S, Béna F, Fort A, Neerman-Arbez M. Developmental expression and organisation of fibrinogen genes in the zebrafish. Thromb Haemost 2012;107(01):158-166

29 Huang W, Sherman BT, Lempicki RA. Systematic and integrative analysis of large gene lists using DAVID bioinformatics resources. Nat Protoc 2009;4(01):44-57

30 Huang W, Sherman BT, Lempicki RA. Bioinformatics enrichment tools: paths toward the comprehensive functional analysis of large gene lists. Nucleic Acids Res 2009;37(01):1-13

31 Jung M-L, Contreras J-M, Morice C, Simon J-M, Didier B, Langer T. The Prestwick Chemical Library ${ }^{\circledR}$ : a valuable tool for screening. Available at: http://www.prestwickchemical.com/pdf/2018-pcla-valuable-tool.pdf. Accessed March 30, 2020

32 Havercroft JC, Quinlan RA, Gull K. Binding of parbendazole to tubulin and its influence on microtubules in tissue-culture cells as revealed by immunofluorescence microscopy. J Cell Sci 1981; 49:195-204

33 Vasquez RJ, Howell B, Yvon AM, Wadsworth P, Cassimeris L. Nanomolar concentrations of nocodazole alter microtubule dynamic instability in vivo and in vitro. Mol Biol Cell 1997;8(06): 973-985

34 Giannattasio C, Cattaneo BM, Seravalle G, et al. Alpha 1-blocking properties of carvedilol during acute and chronic administration. J Cardiovasc Pharmacol 1992;19(Suppl 1):S18-S22

35 Chekman IS. Experimental studies of the mechanism of action of reserpine [in Russian]. Biull Eksp Biol Med 1972;73(03):59-61

36 Nicodeme E, Nicaud M, Issandou M. Retinoids stimulate fibrinogen production both in vitro (hepatocytes) and in vivo. Induction requires activation of the retinoid $X$ receptor. Arterioscler Thromb Vasc Biol 1995;15(10):1660-1667

37 Lin H-F, Traver D, Zhu H, et al. Analysis of thrombocyte development in CD41-GFP transgenic zebrafish. Blood 2005;106(12): 3803-3810

38 Yamashita R, Fujiwara Y, Ikari K, et al. Extracellular proteome of human hepatoma cell, HepG2 analyzed using two-dimensional liquid chromatography coupled with tandem mass spectrometry. Mol Cell Biochem 2007;298(1-2):83-92

39 Dally N, Hoffman R, Haddad N, Sarig G, Rowe JM, Brenner B. Predictive factors of bleeding and thrombosis during induction therapy in acute promyelocytic leukemia-a single center experience in 34 patients. Thromb Res 2005;116(02):109-114

40 Falanga A, Iacoviello L, Evangelista V, et al. Loss of blast cell procoagulant activity and improvement of hemostatic variables in patients with acute promyelocytic leukemia administered alltrans-retinoic acid. Blood 1995;86(03):1072-1081 
41 Torromeo C, Latagliata R, Avvisati G, Petti MC, Mandelli F. Intraventricular thrombosis during all-trans retinoic acid treatment in acute promyelocytic leukemia. Leukemia 2001;15(08):1311-1313

42 Escudier SM, Kantarjian HM, Estey EH. Thrombosis in patients with acute promyelocytic leukemia treated with and without alltrans retinoic acid. Leuk Lymphoma 1996;20(5-6):435-439

43 Losada R, Espinosa E, Hernández C, Dorticos E, Hernández P. Thrombocytosis in patients with acute promyelocytic leukaemia during alltrans retinoic acid treatment. Br J Haematol 1996;95(04):704-705

44 Zhou B, Pan Y, Hu Z, et al. All-trans-retinoic acid ameliorated high fat diet-induced atherosclerosis in rabbits by inhibiting platelet activation and inflammation. J Biomed Biotechnol 2012; 2012:259693

45 Luo Q, Wei G, Wang X, et al. All-trans retinoic acid impairs platelet function and thrombus formation and inhibits protein kinase CßI/ $\delta$ phosphorylation. Thromb Haemost 2019;119(10):1655-1664

46 Ashton RE, Andre P, Lowe NJ, Whitefield M. Anthralin: historical and current perspectives. J Am Acad Dermatol 1983;9(02):173-192
47 Vanizor Kural B, Orem A, Cimşit G, Uydu HA, Yandi YE, Alver A. Plasma homocysteine and its relationships with atherothrombotic markers in psoriatic patients. Clin Chim Acta 2003;332(1-2):23-30

48 Danik JS, Paré G, Chasman DI, et al. Novel loci, including those related to Crohn disease, psoriasis, and inflammation, identified in a genome-wide association study of fibrinogen in 17686 women: the Women's Genome Health Study. Circ Cardiovasc Genet 2009;2(02):134-141

49 Schopf RE, Weber H, Morsches B. Enhanced procoagulant activity of mononuclear leukocytes in patients with atopic dermatitis and psoriasis. Arch Dermatol Res 1993;285(05):305-309

50 Le Fourn V, Park S, Jang I, et al. Large protein complexes retained in the ER are dislocated by non-COPII vesicles and degraded by selective autophagy. Cell Mol Life Sci 2013;70 (11):1985-2002

51 Pushpakom S, Iorio F, Eyers PA, et al. Drug repurposing: progress, challenges and recommendations. Nat Rev Drug Discov 2019;18 (01):41-58 